\title{
Valuing Collaboration and Collaborators
}

\author{
Jennifer Larsen, MD, Vice Chancellor for Research \\ University of Nebraska Medical Center
}

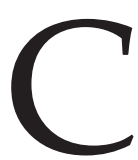

ollaboration and Team Science

The time required to move an innovation from the laboratory and into practice is measured in decades and often leads to failure (1). NIH created the National Center for Advanced Therapeutics https://ncats.nih.gov/programs to house the Clinical and Translational Science Awards Program and other initiatives intended to propel new ways to reduce this time, in part, by creating better 'hand-offs' between laboratory/bench scientists, clinician-scientists, and community health practitioners. Improved translation of research is just one strategy to move biomedical advances more quickly from concept to practice. Many problems we have yet to solve in and outside of the biomedical arena are complex. They require larger teams with specialized expertise to solve them, particularly to acquire and analyze data sets. This collaborative activity is often called Team Science. The research suggests inclusive teams where all members are valued improves the profile of the research (2).

Why Value Collaboration?

There are many reasons to embrace collaboration - to better compete in Team Science, as noted above, is the first. Another reason individual investigators should value collaboration is that collaborative grants and manuscripts often fare better in review, and manuscripts with more collaboration, have a higher citation index (3). Collaboration is also a strategy to achieve independence for early career scientists. By working in collaborative research teams, they will learn from and be mentored by a wider variety of faculty as they develop and acquire pilot data for independent funding. They will also learn to function within and possibly better lead a team in the future. Being included on other grants is also a strategy to bridge funding "gaps", which occurs more often as funding is more competitive. Some promising investigators who don't have a team to support them are KU MASC 2018 Research Retreat dropping out because they don't see a light at the end of the tunnel, particularly if they think success only means a straight line to independent funding and they aren't are on it.

In Team Science, highly technical expertise is not only required but critical. These team members with expertise in biostatistics, biomedical informatics, and use of high-end instrumentation, will predominantly or always be collaborators. An environment that values these team members will be more likely to keep them, for they are highly sought after and can easily leave and find other teams and institutions to join that do.

Community members are also required for many teams, particularly those working on new solutions for community based implementation, as well as health services, health outcomes and quality improvement projects. These members serve in many roles, as coinvestigators, collaborators, consul- 
tants, "cheerleaders", problem solvers, and strategists. They include community leaders and activists as well as community-based health care or public health providers and administrators. Understanding what they want from the collaboration is important as it is likely quite different from technical experts or other team members, but their roles are equally important. Universities should value these collaborators as much as any others because these same community members become higher education and research advocates, and help universities translate the value of research to their state legislators as well as provide training sites for university students.

Knowledge of how to create the best and most effective teams is an important research topic unto itself. Team science and teams are essential to workplaces outside of universities, including hospitals and other health care facilities, manufacturing, and research institutes. Working in a team has other benefits. Finally, being part of a team reduces the stress associated with high impact health care and reduces burnout which now plagues many health care organizations. While research is highly competitive, being a member of a research team may also decrease the stress associated with research careers, although this has not been well studied.

Do institutions value collaboration?

Academic health centers and other universities reward faculty for the activities they value most. Research Faculty learn early what is required to achieve salary increases from their department or college, including promotion and tenure, space assignments, and nominations for awards, internally or externally. Protected time (for research), access to development funds, graduate students and stipends, and choice of education assignments may be equally important to many faculty. With this lens, do institutions reward collaborators and collaboration activities?

Most will admit that promotion guidelines provide a clearer path for independently funded investigators than collaborators, but collaborators in most universities are being promoted more easily than they were in the past. Likelihood is improved when the institutional policies for promotion and tenure more clearly define the criteria required for collaborators, with either a separate path and/or very specific examples. Being awarded tenure can be harder, as this assumes and requires that the faculty member is highly valued by the institution over time. Historically, this requires evidence of independent extramural research funding, but criteria are changing at some institutions when the collaborator has longstanding history of covering their salary, even in the absence of independent funding.

Even when the criteria are clear, roadblocks can occur long before a candidate's packet reaches the university-wide promotion and tenure committee. Chairs, members of division and department promotion and tenure committees, and even mentors are still rooted in the value of more traditional pathways and discourage or reject candidates who are not independent investigators and discourage them from even considering promotion. As a result, the faculty member might not feel valued before they even seek promotion and tenure and begin to "look around". 
Training of the members of promotion and tenure committees is just as important as the criteria and policies for promotion and tenure.

There are other ways to show the institutional value for collaboration. Many University of Nebraska pilot grant programs require evidence of collaboration and many aim at new collaborations. At University of Nebraska Medical Center, we include a metric for collaboration in our Distinguished Scientist Research Awards and encourage collaborators to be nominated for them. We are also working with public affairs to consistently include the names of all collaborators, not just the team leader, in announcements of successful research teams. Separately, we routinely and publically highlight faculty with unique technical expertise, who are often active collaborators. The research space metric used for assignment of space is based on research expenditures, which allows us to value investigators funded by subcontracts as collaborators, as well as those funded as principal investigators by assignment of space.

Yet "value" is defined in the eye of the beholder. Most faculty collaborators express the desire to be valued like other faculty members by traditional mechanisms such as promotion, tenure, and salary. But individual faculty might value something else more. Thus, to demonstrate value to an individual faculty member with a particular skill consider asking them what they value most and you might find they are looking for funds to develop a new technique, to travel to a specific conference, or to relocate their office or laboratory in relation to others. When trying to demonstrate that an individual is valued, consider asking them what they value most first.

The institutional $\mathrm{F} \& \mathrm{~A}$ rate is negotiated with the federal government to reimburse an institution for the true costs of providing infrastructure to support organized research activity. Within our university, all colleges and institutes receive a distribution of the $\mathrm{F} \& \mathrm{~A}$ generated by their faculty's research because those units often share the financial and administrative burden of supporting research, including regulatory compliance, physical research facilities, and grant administration. Some but not all Centers receive $\mathrm{F} \& \mathrm{~A}$ distribution for the same reason. Some universities and institutions also share returned F \& A with the principal investigator, particularly if they are expected to pay for resources such as research space, occupational health screening or other regulatory activities. This often brings up the question of whether F \& A should be shared among the units of the coinvestigators in another college or unit just as the F \& A is distributed to coinvestigators on grants administered at another institution. There is no one answer or strategy on how or if distribution of $\mathrm{F} \& \mathrm{~A}$ demonstrates the value of collaboration. Institutions must first decide how F \& A is spent before deciding if it is warranted and what scheme is most fair and easily administered.

\section{Valuing collaboration may} depend on the type of collaborator.

\section{Core directors}

Service centers, or research "cores", that bill federal grants, must meet all state and federal guidelines. Some cores work regularly with commercial and even international 
clients and develop into independent, "start-up" businesses that require no institutional support. Cores that function within the university and are predominantly focused on serving the needs of investigators at their home university are more likely to require subsidization to end the year without a deficit. In fact, to meet federal guidelines for a service center, any "profit" (excess revenues over costs) must be rolled over into the operations for the following year and taken into consideration when setting the fee schedule going forward.

We expect a lot from core directors. Core directors must have the technical skills to understand the applications and approaches for using the technologies they manage and be able to apply them to a variety of types of research. They must be familiar with up and coming technologies to understand when they are becoming obsolete. They are expected to have or acquire substantial financial skills to resource existing and any new technologies, develop and administer a budget, and set fair prices to meet their budget for the financial operation of service centers. They must know all relevant federal guidelines and when working with international customers, export control regulations, as well. Most importantly, they must have excellent communication and problem-solving skills to hire and retain the best employees, address and resolve problems, even when the customer is highly anxious, frustrated, and/or angry, and market their resource and services, to sustain or expand their customer base and achieve financial sustainability. A core director with all those skills is an institutional asset, and often participates as a research collaborator, as well.

But do core directors feel as valued as other faculty or researchers? It should be noted that core directors may or may not be faculty, and may serve as a core director either full or part-time. While salary is the main avenue to show value, promotion and tenure may be much more difficult. To address this, many institutions have developed pathways for promotion of full time core directors. At UNMC there is a supplemental faculty compensation program for faculty whose salary are on grants, but full time core directors are not eligible. Importantly, the incentive should align with the desired goal and placing their salary on grants may or may not be the outcome most desired for the core director. For this reason, we are developing an incentive stipend mechanism for core directors that aligns with meeting and exceeding the expectations of a core director, to include metrics such as expanding customer base or providing access to new technologies or reducing the cost of core operations. However, if retention is the primary goal for a particular core director, it is important to ask them what they most need, as some core directors might prefer a developmental account to create new applications and techniques relevant to their area of expertise.

Biomedical informatics collabo$\underline{\text { rators }}$

Defining biomedical informatics remains difficult as there are many to choose from, but it describes a broad range of techniques and expertise critical for biomedical science data acquisition, transfer, merging, anonymization, analysis, and storage (4). The types of data 
available are both broad and large ('big data"), from laboratory read outs (e.g., omics data), geographic and environmental data, image and video inputs, and electronic, image, and other health data with its own set of privacy and confidentiality rules. Even individual image, video or genetic data can be large, terabytes, which make moving, sharing, analyzing, and storing them more difficult, whether stored on site, or in "cloud-based" or other sponsored repositories.

No university has all the biomedical informatics expertise they need. The field itself is still rapidly growing and changing with the data being acquired and analyzed in new ways such that many, including leaders, are selftaught. Informaticists or biomedical informatics experts often have and use both programming skills and content knowledge for the data they are handling, such as biostatistics, bioimaging, geographic information systems, or public health systems.

These biomedical informatics specialists often bring their unique skills to research teams as "collaborators". Some may also have their own independently funded research program while others serve only as active collaborators, but are required for many large grant programs. As these same experts are often highly desired by many industries, they can ask for and compete for higher salaries, leadership positions or other titles, graduate students, or new resources that clearly demonstrate their value to the institution.

$\underline{\text { Clinician and community collabo- }}$ $\underline{\text { rators }}$

Clinicians are another type of collaborator required for more and more KU MASC 2018 Research Retreat types of research-to help develop research questions or interpret results, acquire patient data or biologic samples, successfully move research into a clinical trial or useful device, or implement it into a real world setting. Community basedand academic clinicians, in turn, also value being part of a team when it is solving a problem they care about to positively change health care outcomes or practice. Yet health providers have less time for research, whether in a traditional clinical practice or an academic health center, even with funding, because of time constraints and practice requirements.

Academic health centers increasingly realize they need to include this type of participation in their compensation model if they value this type of collaboration so clinicians are not penalized for participation, as well. Community based clinicians may not need or want to be on grants where they have to track their effort. Other ways to show value may be nontraditional and depend on where they work. Providers in communities with known workforce shortages may see participating in research as an opportunity for access to trainees who might then learn about that community and consider working or living there long-term. Being part of a rural community means you often have less access to specialists for informal consultation, so that may be one of their goals. Some clinicians need or desire release time from clinical duties by their health system to participate. Some practitioners value access to library services, continuing medical education credits, or becoming an adjunct faculty. Finally, they may want to participate in the discussions around writing or 
presenting the work at regional or national meetings, as well as being a coauthor.

\section{Summary}

Collaboration is integral to most types of biomedical research, and most researchers serve as collaborators during their career trajectory. Some researchers start out as collaborators and grow into a research leader role. Even research leaders will also participate as collaborators on some projects throughout their career. Faculty who serve predominantly as collaborators because of their unique skills essential to many research teams should be equally valued with a clear path to promotion and tenure or what other recognition or rewards might be needed to show them they are valued by the institution. This may require a change in culture at the promotion and tenure committee or within the faculty at large. In some cases, new mechanisms for reward specific to the type of collaborator or changes in institutional policies and programs will be required. Finally, as team science grows, institutions not only need to create a culture that values collaboration, but teach the next generation how to function effectively within a team as this skill will be as important to their future success as grantsmanship or any technical skill they are learning today.

\section{Citations}

1. Collins, FS, Reengineering Translational Science: The Time Is Right. Science Translational Medicine 3: 90, 2017

2. Bennett, LM, and Gadlin, H, Collaboration and Team Science: From Theory to Practice J Investig Med. 60: 768, 2012.

3. Parish AJ, Boyack KW, Ioannidis JPA. Dynamics of co-authorship and productivity across different fields of scientific research. PLoS One. 13: 2018

4. Bernstam, EV, Smith, JW, Johnson, TR What is biomedical informatics? J Biomed Inform. 2010 Feb; 43(1): 104. 